\title{
Group multi-criteria decision making using intuitionistic multi-fuzzy sets
}

\author{
Sujit Das ${ }^{1}$, Mohuya B Kar ${ }^{2}$ and Samarjit $\mathrm{Kar}^{3^{*}}$
}

\author{
* Correspondence: \\ kar_s_k@yahoo.com \\ ${ }^{3}$ Department of Mathematics, \\ National Institute of Technology, \\ Durgapur, West Bengal 713209, \\ India \\ Full list of author information is \\ available at the end of the article
}

\begin{abstract}
In this paper we propose an efficient approach for group multi-criteria decision making (MCDM) based on intuitionistic multi-fuzzy set (IMFS). First we construct intuitionistic multi-fuzzy matrices for decision makers with respect to the criteria (attributes) of the alternatives. Based on intuitionistic multi-fuzzy matrices, we construct the aggregated intuitionistic multi-fuzzy matrix using the proposed intuitionistic multi-fuzzy weighted averaging (IMFWA) operator. Then we use Hamming distance and Euclidean distance measurements in the context of IMFS between the aggregated matrix and the specified sample matrix to reach the optimal decision. This paper also presents score function and accuracy function of IMFS with an application to MCDM. Finally, a real-life case study related to heart disease diagnosis problem is provided to illustrate the advantage of the proposed approach.
\end{abstract}

Keywords: Group MCDM; Intuitionistic multi-fuzzy set; Intuitionistic multi-fuzzy weighted averaging operator; Hamming distance; Euclidean distance; Score function; Accuracy function; Medical diagnosis

\section{Introduction}

Intuitionistic fuzzy set (IFS) was introduced by Atanassov [1] and can be shown as a generalization and extension of Zadeh's fuzzy set theory [2]. IFS has emerged as an active research area mainly for solving multiple-criteria decision making problems [3-9] and group decision making (GDM) problems [10-12] where the values of local criteria (attributes) of alternatives and/or their weights are intuitionistic fuzzy values (IFVs). Intuitionistic fuzzy set covers such kind of situations where a human being can express the degree of belonging of an element to a set as well as degree of non-belonging of an element to a set.

A complete account of the development of multi-set theory has been seen in [13]. As a generalization of multi-set, Yager [14] introduced the concept of multi-fuzzy set (MFS). An element of a MFS can occur more than once with possibly the same or different membership values. MFS theory is an extension of theories of fuzzy sets [2], L-fuzzy sets [15], and intuitionistic fuzzy sets [16]. Group multi-criteria decision making requires certain methods to aggregate the opinions provided by different experts. Yager [17] proposed an interesting and well-grounded approach, namely the ordered weighted averaging (OWA) [17], which enabled aggregation of the variables in terms of their order in the set.

(c) 2013 Das et al.; licensee Springer. This is an open access article distributed under the terms of the Creative Commons Attribution License (http://creativecommons.org/licenses/by/2.0), which permits unrestricted use, distribution, and reproduction in any medium, provided the original work is properly cited. 
Most of the human reasoning involves linguistic variables whose values are words rather than numbers to express a situation in a better way in an uncertain environment. Fuzzy sets act as the basis of these linguistic variables. But in some situations like decision making problems (such as medical diagnosis, sales analysis, marketing, etc.), the description by a linguistic variable in terms of membership function only is not adequate. In many cases, there is an obvious chance of an appearance of a non-null complement. IFS can be used in this context as a proper tool for representing both membership and non-membership of an element to a concept [18]. Again there are situations where each element has different membership values. In such situations, an intuitionistic multi-fuzzy set (IMFS) is more adequate. Here we present IMFS as a tool for representing such situations linguistically.

The aim of this paper is to combine the concept of intuitionistic fuzzy set and multifuzzy set to produce an intuitionistic multi-fuzzy set and apply this to group MCDM problems. In group MCDM, a group of decision makers provide their opinions based on their own observations and intuitions over a set of attributes. The individual opinions are finally converted to a collective opinion using some suitable procedure. This article introduces IMFSs, basic operations on IMFSs, and application of IMFSs to MCDM problems by constructing a suitable algorithmic approach. Here we also present score function and accuracy function in the context of IMFS with an application to MCDM. In the proposed approach, we first construct intuitionistic multi-fuzzy matrices (IMFMs) for decision makers. Decision makers provide their opinions in terms of linguistic scales at different time instances which are represented by IMFMs. Next we construct the aggregated intuitionistic multi-fuzzy matrix which reflects the combined opinions of all decision makers using our proposed intuitionistic multi-fuzzy weighted averaging (IMFWA) operator. IMFWA can be treated as an extension of intuitionistic weighted arithmetic mean (IWAM) [19]. Then this study uses IMFSbased normalized Hamming distance and normalized Euclidean distance to find out the final outcome. Finally, this study degenerates the individual experts' opinions (IMFS) into IFS and compares the final outcome. This approach is illustrated using a suitable case study concerned with medical disease diagnosis.

The rest of this article is organized as follows: The second section presents the preliminaries about IFS and some operations on IFS. The third section includes a brief description about MFS, IMFS, and few operations on IMFS including IMFWA operator and distance measurements. The fourth section includes the proposed algorithmic approach, followed by a case study in the fifth section. Finally, the paper is concluded in the sixth section.

\section{Intuitionistic fuzzy sets and some operations}

In this section, we briefly review the definition of IFS, its operations, and comparisons.

\section{Definition of IFS and IFV with necessary conditions}

Definition 1 Intuitionistic fuzzy sets were introduced by Atanassov [1]. Let $X$ be a universal set and $A \subset X$. An intuitionistic fuzzy set is a set

$$
A=\left\{x, \mu_{A}(x), v_{A}(x): x \in X\right\}
$$


where the functions $\mu_{A}: X \rightarrow[0,1]$ and $\nu_{A}: X \rightarrow[0,1]$ reflect the degree of membership and non-membership of the element $x$ from $X$ to set $A$, respectively, and for every $x \in$ $X$, it is also fulfilled that

$$
0 \leq \mu_{A}(x)+v_{A}(x) \leq 1 .
$$

The function $\pi_{A}: X \rightarrow[0,1]$, given by $\pi_{A}(x)=1-\mu_{A}(x)-v_{A}(x), x \in X$, defines the degree of uncertainty of the membership of the element $x$ to set $A$ known as hesitation function. For a fixed $x \in X$, an object $\left\{\mu_{A}(x), v_{A}(x)\right\}$ is usually called IFV or intuitionistic fuzzy number (IFN).

\section{Description of two basic operations using intuitionistic fuzzy sets Addition $\oplus$ and multiplication $\otimes$ of IFSs}

The operations of addition $\oplus$ and multiplication $\otimes$ on intuitionistic fuzzy values were defined by Atanassov [19] as follows: Let $A=\left(\mu_{A}, v_{A}\right)$ and $B=\left(\mu_{B}, v_{B}\right)$ be IFVs, then

$$
\begin{aligned}
& A \oplus B=\left(\mu_{A}+\mu_{B}-\mu_{A} \cdot \mu_{B}, v_{A} \cdot v_{B}\right) \\
& A \otimes B=\left(\mu_{A} \cdot \mu_{B}, v_{A}+v_{B}-v_{A} \cdot v_{B}\right) .
\end{aligned}
$$

These operations were constructed in such a way that they produce IFVs since it is easy to prove that $0 \leq \mu_{A}+\mu_{B}-\mu_{A} \cdot \mu_{B}+v_{A} \cdot v_{B} \leq 1$ and $0 \leq \mu_{A} \cdot \mu_{B}+v_{A}+v_{B}-v_{A} \cdot v_{B} \leq 1$.

\section{Multiplication $\otimes$ of IFS with real values}

The addition and multiplication of IFSs with real values were obtained using expressions (1) and (2) in [20]. For any integer $n, n A=\left\{1-\left(1-\mu_{A}\right)^{n}, v_{A}^{n}\right\}$ and $A^{n}=\left\{\mu_{A}^{n}, 1-\left(1-v_{A}\right)^{n}\right\}$. It was proven later that these operations produce IFVs not only for integer $n$ but also for all real values $\lambda>0$, i.e.,

$$
\begin{aligned}
& \lambda A=\left\{1-\left(1-\mu_{A}\right)^{\lambda}, v_{A}^{\lambda}\right\} \\
& A^{\lambda}=\left\{\mu_{A}^{\lambda}, 1-\left(1-v_{A}\right)^{\lambda}\right\} .
\end{aligned}
$$

Definition 2 Intuitionistic weighted arithmetic mean: The IWAM can be obtained using expressions (1) and (3) as follows:

$$
\text { IWAM }=w_{1} A_{1} \oplus w_{2} A_{2} \oplus \ldots \oplus w_{n} A_{n}=\left\{1-\prod_{i=1}^{n}\left(1-\mu_{A_{i}}\right)^{w_{i}}, \coprod_{i=1}^{n} v_{A_{i}}^{w_{i}}\right\}
$$

where $0 \leq$ wi $\leq 1$ and $\sum_{i=1}^{n} w_{i}=1$.

This aggregating operator provides IFVs and is currently the most popular in the solution of MCDM problems in the intuitionistic fuzzy setting.

\section{Comparison of IFSs}

An important problem is the comparison of IFVs. Chen and Tan [3] proposed to use the score function (or net membership) as $S(x)=\mu(x)-v(x)$ where $x$ is an IFV. Hong and Choi [4] in addition to the above score function introduced the accuracy function as $H(x)=\mu(x)+v(x)$. Xu [21] used both the functions $S$ and $H$ to construct order relations between any pair of intuitionistic fuzzy values ( $x$ and $y$ ) as follows: 
If $(S(x)>S(y))$, then $x>y$;

If $(S(x)==S(y))$, then

(1) if $(H(x)==H(y))$, then $x=y$;

(2) if $(H(x)<H(y))$, then $x<y$.

\section{Intuitionistic multi-fuzzy set and some operations}

This section briefly describes multi-fuzzy set, intuitionistic multi-fuzzy set, and a few operations on IMFS including IMFWA operator and distance measurements.

\section{MFS and IMFS}

Definition 3 Let $k$ be a positive integer and $U$ be a universal set. A multi-fuzzy set $A$ over $U$ is a set of ordered sequences $A=\left\{x /\left(\mu_{A}^{1}(x), \mu_{A}^{2}(x), \ldots, \mu_{A}^{i}(x), \ldots, \mu_{A}^{k}(x)\right): x \in U\right\}$ where $\mu_{A}^{i} \in P(U), \quad i=1,2, \ldots, k$.

The function $\mu_{A}=\left(\mu_{A}^{1}, \mu_{A}^{2}, \ldots, \mu_{A}^{k}\right)$ is called a multi-membership function of multifuzzy set $A$, and $k$ is called the dimension of multi-fuzzy set $A$.

Definition 4 An intuitionistic multi-fuzzy set $A$ is defined by $A=$ $\left\{x /\left(\mu_{A}^{1}(x), \mu_{A}^{2}(x), \ldots, \mu_{A}^{k}(x)\right),\left(v_{A}^{1}(x), v_{A}^{2}(x), \ldots, v_{A}^{k}(x)\right): x \in U\right\}$, where $0 \leq \mu_{A}^{i}(x)+v_{A}^{i}(x) \leq$ $1, \pi_{A}^{i}(x)=1-\left(\mu_{A}^{i}(x)+v_{A}^{i}(x)\right)$ for every $x \in U$ and $i=1,2, \ldots, k$ where $k$ is a positive integer and $U$ is a universal set.

For a fixed $x \in U$, an object $\left\{\mu_{A}^{i}(x), v_{A}^{i}(x)\right\}$ is usually called an intuitionistic multi-fuzzy value (IMFV) or intuitionistic multi-fuzzy number (IMFN) where $i=1,2, \ldots, k$ and $k>0$.

Remark 1 If we arrange the membership sequences in decreasing order, then the corresponding non-membership sequence may not be in decreasing or increasing order.

Remark 2 The sequences of membership functions and non-membership functions only have $k$ terms (finite number of terms), and $k$ is known as the dimension of $A$.

\section{Some useful operations on IMFS}

For any two intuitionistic multi-fuzzy sets $A$ and $B$ of dimension $k$, the following operations can be defined. Let

$$
\begin{aligned}
& A=\left\{x /\left(\mu_{A}^{1}(x), \mu_{A}^{2}(x), \ldots, \mu_{A}^{k}(x)\right),\left(v_{A}^{1}(x), v_{A}^{2}(x), \ldots, v_{A}^{k}(x)\right): x \in U\right\} \\
& B=\left\{x /\left(\mu_{B}^{1}(x), \mu_{B}^{2}(x), \ldots, \mu_{B}^{k}(x)\right),\left(v_{B}^{1}(x), v_{B}^{2}(x), \ldots, v_{B}^{k}(x)\right): x \in U\right\}
\end{aligned}
$$

\section{Addition of IMFS $(A \oplus B)$}

In $A \oplus B$, the membership and non-membership values are obtained as follows:

$$
\mu_{A \oplus B}^{j}=\mu_{A}^{j}(x)+\mu_{B}^{j}(x)-\mu_{A}^{j}(x) \cdot \mu_{B}^{j}(x), v_{A \oplus B}^{j}=v_{A}^{j}(x) \cdot v_{B}^{j}(x), j=1,2, \ldots, k, x \in U .
$$

\section{Multiplication of IMFS $(A \otimes B)$}

In $A \otimes B$, the membership and non-membership values are obtained as follows:

$$
\begin{aligned}
& \mu_{A \otimes B}^{j}=\mu_{A}^{j}(x) \cdot \mu_{B}^{j}(x), \\
& v_{A}^{j} \otimes B \\
& j=v_{A}^{j}(x)+v_{B}^{j}(x)-v_{A}^{j}(x) \cdot v_{B}^{j}(x), \\
& j=1,2, \ldots, k, x \in U .
\end{aligned}
$$

Example 1 Let $A$ and $B$ be two intuitionistic multi-fuzzy sets of dimension 3 defined as follows: 


$$
\begin{aligned}
& A=\{(0.7,0.6,0.5),(0.1,0.2,0.4)\} \\
& B=\{(0.4,0.4,0.3),(0.3,0.4,0.6)\}
\end{aligned}
$$

The addition $(A \oplus B)$ and multiplication $(A \otimes B)$ of $A$ and $B$ can be obtained by

$$
\begin{aligned}
& A \oplus B=\{(0.82,0.76,0.65),(0.03,0.08,0.24)\}, \\
& A \otimes B=\{(0.28,0.24,0.15),(0.37,0.52,0.76)\} .
\end{aligned}
$$

\section{Multiplication of IMFS A with real value}

Multiplication of IMFS $A$ of dimension $k$ with real value $\lambda>0$ can be defined as

$$
\begin{aligned}
& \lambda A=\left\{1-\left(1-\mu_{A}^{j}\right)^{\lambda},\left(v_{A}^{j}\right)^{\lambda}\right\}, \\
& A^{\lambda}=\left\{\left(\mu_{A}^{j}\right)^{\lambda}, 1-\left(1-v_{A}^{j}\right)^{\lambda}\right\}, \\
& j=1,2, \ldots, k, x \in U .
\end{aligned}
$$

Example 2 Let $A$ be an intuitionistic multi-fuzzy set of dimension 3 where $A$ is defined as follows and $\lambda=0.5$.

$$
A=\{(0.7,0.6,0.5),(0.1,0.2,0.4)\},
$$

Now $\lambda A=(0.45,0.37,0.29)(0.32,0.45,0.63), A^{\lambda}=(0.84,0.77,0.71)(0.05,0.11,0.23)$.

\section{Proposed IMFWA operator}

Definition 5 Intuitionistic multi-fuzzy weighted arithmetic operator can be defined by

$$
\begin{aligned}
& \text { IMFWA }=w_{1} A_{1} \oplus w_{2} A_{2} \oplus \ldots \oplus w_{n} A_{n} \\
& =\left\{1-\prod_{i=1}^{n} \prod_{j=1}^{k}\left(1-\mu_{A_{i}}^{j}\right)^{w_{i}}, \prod_{i=1}^{n} \prod_{j=1}^{k}\left(v_{A_{i}}^{j}\right)^{w_{i}}, \prod_{i=1}^{n} \prod_{j=1}^{k}\left(1-\mu_{A_{i}}^{j}\right)^{w_{i}}-\prod_{i=1}^{n} \prod_{j=1}^{k}\left(v_{A_{i}}^{j}\right)^{w_{i}}\right\}
\end{aligned}
$$

where $A_{1}, A_{2}, \ldots, A_{n}$ are IMFS of dimension $k$ and $w_{1}, w_{2}, \ldots, w_{n}$ are their weights where $\sum_{i=1}^{n} w_{i}=1$ and $w_{i} \in[0,1]$.

Example 3 Let $A_{1}$ and $A_{2}$ be two intuitionistic multi-fuzzy sets of dimension 3, and weights $w_{1}=0.3$ and $w_{2}=0.7$ are assigned to them. Let

$$
\begin{aligned}
& A_{1}=\{(0.7,0.6,0.5),(0.1,0.2,0.4)\}, \\
& A_{2}=\{(0.4,0.4,0.3),(0.3,0.4,0.6)\} .
\end{aligned}
$$

The aggregated IMFWA is computed as

$$
\begin{aligned}
& \operatorname{IMFWA}\left(A_{1}, A_{2}\right)=w_{1} A_{1} \oplus w_{2} A_{2}= \\
& 1-\left\{(1-0.7)^{0.3}(1-0.6)^{0.3}(1-0.5)^{0.3}(1-0.4)^{0.7}(1-0.4)^{0.7}(1-0.3)^{0.7}\right\}, \\
& \left\{(0.1)^{0.3}(0.2)^{0.3}(0.4)^{0.3}(0.3)^{0.7}(0.4)^{0.7}(0.6)^{0.7}\right\}=\{0.84,0.04\} .
\end{aligned}
$$

Definition of score function $S(x), x \in U$ and accuracy function $H(x), x \in U$ for IMFSs

Definition 6 If $x$ is an IMFV where $x=\left\{\left(\mu_{x}^{1}, \mu_{x}^{2}, \ldots, \mu_{x}^{k}\right),\left(v_{x}^{1}, v_{x}^{2}, \ldots, v_{x}^{k}\right)\right\}$, the score function can be defined as 
Table 1 IMFVs and choice values

\begin{tabular}{lllllll}
\hline U/E & $\boldsymbol{e}_{\mathbf{1}}$ & $\boldsymbol{e}_{\mathbf{2}}$ & $\boldsymbol{e}_{\mathbf{3}}$ & $\boldsymbol{e}_{\mathbf{4}}$ & $\boldsymbol{e}_{\mathbf{5}}$ & Choice value \\
\hline$h_{1}$ & $(0.3,0.7,0.5)$ & $(0.4,0.3,0.4)$ & $(0.1,0.2,0.0)$ & $(0.5,0.6,0.7)$ & $(0.4,0.3,0.4)$ & $(1.7,2.1,2.0)$ \\
& $(0.2,0.1,0.4)$ & $(0.3,0.6,0.4)$ & $(0.7,0.7,0.8)$ & $(0.4,0.3,0.2)$ & $(0.6,0.4,0.4)$ & $(2.2,2.1,2.2)$ \\
$h_{2}$ & $(0.4,0.3,0.5)$ & $(0.7,0.6,0.8)$ & $(0.6,0.5,0.4)$ & $(0.3,0.6,0.2)$ & $(0.4,0.7,0.5)$ & $(2.4,2.7,2.4)$ \\
& $(0.5,0.4,0.4)$ & $(0.2,0.2,0.1)$ & $(0.3,0.3,0.4)$ & $(0.7,0.3,0.7)$ & $(0.1,0.2,0.3)$ & $(1.8,1.4,1.9)$ \\
$h_{3}$ & $(0.1,0.2,0.1)$ & $(0.3,0.2,0.1)$ & $(0.8,0.7,0.8)$ & $(0.3,0.2,0.2)$ & $(0.4,0.3,0.2)$ & $(1.9,1.6,1.4)$ \\
& $(0.7,0.6,0.9)$ & $(0.6,0.0,0.7)$ & $(0.0,0.1,0.1)$ & $(0.6,0.7,0.6)$ & $(0.4,0.7,0.7)$ & $(2.3,2.1,3.0)$ \\
$h_{4}$ & $(0.5,0.4,0.5)$ & $(0.4,0.3,0.4)$ & $(0.2,0.1,0.0)$ & $(0.5,0.6,0.3)$ & $(0.4,0.5,0.4)$ & $(2.0,1.9,1.6)$ \\
& $(0.4,0.4,0.3)$ & $(0.5,0.3,0.5)$ & $(0.7,0.6,0.7)$ & $(0.4,0.3,0.6)$ & $(0.6,0.4,0.3)$ & $(2.6,2.0,2.4)$ \\
$h_{5}$ & $(0.6,0.2,0.7)$ & $(0.8,0.3,0.4)$ & $(0.3,0.3,0.3)$ & $(0.3,0.4,0.1)$ & $(0.5,0.3,0.4)$ & $(2.5,1.5,1.9)$ \\
& $(0.3,0.4,0.2)$ & $(0.1,0.5,0.4)$ & $(0.6,0.4,0.3)$ & $(0.5,0.5,0.8)$ & $(0.3,0.5,0.5)$ & $(1.8,2.3,2.2)$ \\
$h_{6}$ & $(0.6,0.6,0.8)$ & $(0.4,0.3,0.3)$ & $(0.6,0.4,0.3)$ & $(0.6,0.3,0.2)$ & $(0.3,0.4,0.2)$ & $(2.5,2.0,1.8)$ \\
& $(0.2,0.3,0.1)$ & $(0.5,0.1,0.2)$ & $(0.2,0.4,0.4)$ & $(0.3,0.6,0.4)$ & $(0.3,0.4,0.6)$ & $(1.5,1.8,1.7)$ \\
\hline
\end{tabular}

$$
S(x)=\sum_{j}\left\{\mu^{j}(x)-v^{j}(x)\right\}, j=1,2, \ldots, k
$$

The accuracy function can be defined as

$$
H(x)=\sum_{j}\left\{\mu^{j}(x)+v^{j}(x)\right\}, j=1,2, \ldots, k .
$$

\section{Comparison of IMFS}

If $x$ and $y$ are IMFVs where $x=\left\{\left(\mu_{x}^{1}, \mu_{x}^{2}, \ldots, \mu_{x}^{k}\right),\left(v_{x}^{1}, v_{x}^{2}, \ldots, v_{x}^{k}\right)\right\} \quad$ and $\quad y=$ $\left\{\left(\mu_{y}^{1}, \mu_{y}^{2}, \ldots, \mu_{y}^{k}\right),\left(v_{y}^{1}, v_{y}^{2}, \ldots, v_{y}^{k}\right)\right\}$, then $x$ and $y$ can be compared as follows:

Table 2 Score function and choice values

\begin{tabular}{lccc}
\hline $\boldsymbol{U}$ & Choice value & Score value & Accuracy value \\
\hline$h_{1}$ & $(1.7,2.1,2.0)$ & 0.7 & 12.4 \\
& $(2.2,2.1,2.2)$ & & 12.6 \\
$h_{2}$ & $(2.4,2.7,2.4)$ & 2.4 & 12.3 \\
& $(1.8,1.4,1.9)$ & & \\
$h_{3}$ & $(1.9,1.6,1.4)$ & 2.5 & 12.5 \\
& $(2.3,2.1,3.0)$ & & 1.5 \\
$h_{4}$ & $(2.0,1.9,1.6)$ & & 1.2 \\
& $(2.6,2.0,2.4)$ & 1.8 & 1.3 \\
$h_{5}$ & $(2.5,1.5,1.9)$ & & \\
& $(1.8,2.3,2.2)$ & 1.3 & \\
$h_{6}$ & $(2.5,2.0,1.8)$ & & \\
& $(1.5,1.8,1.7)$ & & \\
\hline
\end{tabular}


if $(S(x)>S(y))$, then $x>y$;

if $(S(x)==S(y))$, then

(1) if $(H(x)==H(y))$, then $x=y$;

(2) if $(H(x)<H(y))$, then $x<y$.

Example 4 Let $x=\{(0.7,0.6,0.5),(0.1,0.2,0.4)\}$ and $y=\{(0.4,0.4,0.3),(0.3,0.4,0.6)\}$. Now

$$
\begin{aligned}
S(x) & =\{(0.7-0.1)+(0.6-0.2)+(0.5-0.4)\}=1.1 \\
S(y) & =\{(0.4-0.3)+(0.4-0.4)+(0.3-0.6)\}=-0.2 \\
H(x) & =\{(0.7+0.1)+(0.6+0.2)+(0.5+0.4)\}=2.5 \\
H(y) & =\{(0.4+0.3)+(0.4+0.4)+(0.3+0.6)\}=2.4
\end{aligned}
$$

Since $S(x)>S(y)$, therefore $x>y$.

Example 5 Suppose $U=\left\{h_{1}, h_{2}, h_{3}, h_{4}, h_{5}, h_{6}\right\}$ is a set of six houses (alternatives) and $E=\left\{e_{1}, e_{2}, e_{3}, e_{4}, e_{5}\right\}$ is a set of five parameters (criteria). Mr. X wants to buy a house based on the intuitionistic multi-fuzzy values provided for all the parameters of the given houses which are shown in Table 1. Intuitionistic multi-fuzzy choice values $\left(\mu_{c}^{i}, v_{c}^{i}\right)$ are computed as $\mu_{c}^{i}=\sum_{j=1}^{n} \mu^{i}\left(x_{j}\right), i=1,2, \ldots, k$ and $v_{c}^{i}=\sum_{j=1}^{n} v^{i}\left(x_{j}\right), i=1,2, \ldots, k$

(Table 1). Table 2 shows the score function and accuracy function measurements with respect to the intuitionistic multi-fuzzy choice values. As per comparisons of IMFVs, the maximum score value gives the optimal alternatives, i.e., house $h_{3}$. Therefore, Mr. $\mathrm{X}$ will go for house $h_{3}$. This example shows the application of score function and accuracy functions in multi-criteria decision making problems.

\section{Distance measurements over IMFSs}

Definition 7 Hamming distance $d^{\mathrm{H}}(A, B)$ between two IMFS, $A$ and $B$, in $U=\left(x_{1}, x_{2}, \ldots, x_{n}\right)$ of dimension $k$ can be defined as follows:

$$
d^{\mathrm{H}}(A, B)=\frac{1}{2 k}\left(\sum_{j=1}^{n} \sum_{i=1}^{k}\left(\left|\mu_{A}^{i}\left(x_{j}\right)-\mu_{B}^{i}\left(x_{j}\right)\right|+\left|v_{A}^{i}\left(x_{j}\right)-v_{B}^{i}\left(x_{j}\right)\right|+\left|\pi_{A}^{i}\left(x_{j}\right)-\pi_{B}^{i}\left(x_{j}\right)\right|\right)\right), x \in U .
$$

Normalized Hamming distance $l^{\mathrm{H}}(A, B)$ between two IMFS, $A$ and $B$, in $U=\left(x_{1}, x_{2}, \ldots, x_{n}\right)$ of dimension $k$ can be defined as

$$
l^{\mathrm{H}}(A, B)=\frac{1}{2 n k}\left(\sum_{j=1}^{n} \sum_{i=1}^{k}\left(\left|\mu_{A}^{i}\left(x_{j}\right)-\mu_{B}^{i}\left(x_{j}\right)\right|+\left|v_{A}^{i}\left(x_{j}\right)-v_{B}^{i}\left(x_{j}\right)\right|+\left|\pi_{A}^{i}\left(x_{j}\right)-\pi_{B}^{i}\left(x_{j}\right)\right|\right)\right), x \in U .
$$

Example 6 Let $A$ and $B$ be two IMFSs of dimension 3.

$$
A=\{(0.7,0.6,0.5),(0.1,0.2,0.4)\}, B=\{(0.4,0.4,0.3),(0.3,0.4,0.6)\} .
$$

The Hamming distance $d^{\mathrm{H}}(A, B)$ can be computed as

$$
\begin{aligned}
d^{\mathrm{H}}(A, B)= & \frac{1}{2}\{(|0.7-0.4|+|0.1-0.3|+|0.2-0.3|)+(|0.6-0.4|+|0.2-0.4|+|0.2-0.2|) \\
& +(|0.5-0.3|+|0.4-0.6|+|0.1-0.1|)\}=0.7
\end{aligned}
$$


The normalized Hamming $l^{\mathrm{H}}(A, B)$ distance can be computed as

$$
\begin{aligned}
l^{\mathrm{H}}(A, B)= & \frac{1}{2 \times 3}\{(|0.7-0.4|+|0.1-0.3|+|0.2-0.3|)+(|0.6-0.4|+|0.2-0.4|+|0.2-0.2|) \\
& +(|0.5-0.3|+|0.4-0.6|+|0.1-0.1|)\}=0.23 .
\end{aligned}
$$

Definition 8 Euclidean distance $d^{\mathrm{E}}(A, B)$ between two IMFS, $A$ and $B$, in $U=\left(x_{1}, x_{2}, \ldots, x_{n}\right)$ of dimension $k$ can be defined as follows:

$$
d^{\mathrm{E}}(A, B)=\left(\frac{1}{2 k} \sum_{j=1}^{n} \sum_{i=1}^{k}\left(\mu_{A}^{i}\left(x_{j}\right)-\mu_{B}^{i}\left(x_{j}\right)\right)^{2}+\left(v_{A}^{i}\left(x_{j}\right)-v_{B}^{i}\left(x_{j}\right)\right)^{2}+\left(\pi_{A}^{i}\left(x_{j}\right)-\pi_{B}^{i}\left(x_{j}\right)\right)^{2}\right)^{\frac{1}{2}}, x \in U .
$$

The normalized Euclidean distance $l^{\mathrm{E}}(A, B)$ between two IMFS, $A$ and $B$, in $U=\left(x_{1}\right.$, $\left.x_{2}, \ldots, x_{n}\right)$ of dimension $k$ can be defined as

$$
l^{\mathrm{E}}(A, B)=\left(\frac{1}{2 n k} \sum_{j=1}^{n} \sum_{i=1}^{k}\left(\mu_{A}^{i}\left(x_{j}\right)-\mu_{B}^{i}\left(x_{j}\right)\right)^{2}+\left(v_{A}^{i}\left(x_{j}\right)-v_{B}^{i}\left(x_{j}\right)\right)^{2}+\left(\pi_{A}^{i}\left(x_{j}\right)-\pi_{B}^{i}\left(x_{j}\right)\right)^{2}\right)^{\frac{1}{2}}, x \in U .
$$

Example7 Let $A$ and $B$ be two IMFS of dimension 3. Let

$$
A=\{(0.7,0.6,0.5),(0.1,0.2,0.4)\}, B=\{(0.4,0.4,0.3),(0.3,0.4,0.6)\} .
$$

The Euclidean distance $d^{\mathrm{E}}(A, B)$ can be computed as $d^{\mathrm{E}}(A, B)=\left(\frac{1}{2}\left[\left\{(0.7-0.4)^{2}+(0.1-0.3)^{2}\right.\right.\right.$ $\left.+(0.2-0.3)^{2}\right\}+\left\{(0.6-0.4)^{2}+(0.2-0.4)^{2}+(0.2-0.2)^{2}\right\}+\left\{(0.5-0.3)^{2}+(0.4-0.6)^{2}+\right.$ $\left.\left.\left.(0.1-0.1)^{2}\right\}\right]\right)^{\frac{1}{2}}=0.39$. The normalized Euclidean distance $l^{\mathrm{E}}(A, B)$ can be computed as

$$
\begin{aligned}
l^{\mathrm{E}}(A, B)=\left(\frac { 1 } { 2 \times 3 } \left[\left\{(0.7-0.4)^{2}\right.\right.\right. & \left.+(0.1-0.3)^{2}+(0.2-0.3)^{2}\right\}+\left\{(0.6-0.4)^{2}\right. \\
& \left.+(0.2-0.4)^{2}+(0.2-0.2)^{2}\right\}+\left\{(0.5-0.3)^{2}\right. \\
& \left.\left.\left.+(0.4-0.6)^{2}+(0.1-0.1)^{2}\right\}\right]\right)^{\frac{1}{2}}=0.22 .
\end{aligned}
$$

\section{Presentation of IMFS to IFS degeneration}

An intuitionistic multi-fuzzy set $A=\left\{x /\left(\mu_{A}^{1}(x), \mu_{A}^{2}(x), \ldots, \mu_{A}^{k}(x)\right)\left(v_{A}^{1}(x), v_{A}^{2}(x), \ldots, v_{A}^{k}\right.\right.$

$(x)): x \in U\}$ can be degenerated to an IFS $A^{G}$ where $A^{G}=\left\{x /\left(\mu_{A}^{G}(x),\left(v_{A}^{G}(x)\right): x \in U\right\}\right.$,

$\mu_{A}^{G}(x)=\frac{1}{k} \sum_{i=1}^{k} \mu_{A}^{i}, v_{A}^{G}(x)=\frac{1}{k} \sum_{i=1}^{k} v_{A}^{i}$, and $0 \leq \mu_{A}^{G}(x)+v_{A}^{G}(x) \leq 1, \pi_{A}^{G}(x)=1-\left(\mu_{A}^{G}(x)+v_{A}^{G}(x)\right)$ for every $x \in U$ and $i=1,2, \ldots, k$ where $k$ is a positive integer and $U$ is a universal set.

Example 8 Let $A$ be an IMFS of dimension 3. If $A=\{(0.7,0.6,0.5),(0.1,0.2,0.4)\}$, then IFS $A^{G}$ can be expressed as $A^{G}=\{(0.7+0.6+0.5) / 3,(0.1+0.2+0.4) / 3\}=(0.6,0.2)$.

\section{An algorithm for IMFS-based GDM}

In this section, we develop an algorithm for an intuitionistic multi-fuzzy set-based group decision making method using Hamming distance measurement technique. 
Step 1: Intuitionistic multi-fuzzy matrices are constructed based on the values provided by the decision makers. Assume that the rating of $i$ th alternatives $x_{i}$ with respect to the $j$ th criteria $c_{j}$ given by the $l$ th decision maker $d_{l}$ are expressed in IMFS as $r_{i j}^{(l)}=\left\{\left(\mu_{i j}^{(1 l)}(x), \mu_{i j}^{(2 l)}(x), \ldots, \mu_{i j}^{(k l)}(x)\right),\left(v_{i j}^{(1 l)}(x), v_{i j}^{(2 l)}(x), \ldots, v_{i j}^{(k l)}(x)\right): x \in U\right\}$, where $k$ is the dimension of IMFS. $M, N$, and $L$ are the number of alternatives, criteria, and decision makers, respectively. Hence, a multi-criteria group decision making problem using IMFS can be expressed by the following matrix.

$$
R^{(l)}=\left(r_{i j}^{(l)}\right)_{m \times n}=\left[\begin{array}{cccc}
r_{11}^{(l)} & r_{12}^{(l)} & \cdots & r_{1 n}^{(l)} \\
r_{21}^{(l)} & r_{22}^{(l)} & \cdots & r_{2 n}^{(l)} \\
\vdots & \vdots & \ddots & \vdots \\
r_{m 1}^{(l)} & r_{m 2}^{(l)} & \cdots & r_{m n}^{(l)}
\end{array}\right]
$$

where $l \in L, i \in M, j \in N, m=|M|, n=|N|$, and $r_{i j}^{(l)}=\left\{\left(\mu_{i j}^{(1 l)}(x), \mu_{i j}^{(2 l)}(x), \ldots, \mu_{i j}^{(k l)}(x)\right),\left(v_{i j}^{(1 l)}(x), v_{i j}^{(2 l)}(x), \ldots, v_{i j}^{(k l)}(x)\right): x \in U\right\}$.

Step 2: A weight is provided to each decision maker $d_{l}(l \in L)$ which can be expressed as $w_{i} \in[0,1] \forall i \in L$ where $\sum_{i=1}^{L} w_{i}=1$.

Step 3: An aggregated intuitionistic multi-fuzzy matrix is constructed based on the opinions of the decision makers. Let $R^{(l)}=\left(r_{i j}^{(l)}\right)_{m \times n}$ be an intuitionistic multi-fuzzy matrix of the $l$ th decision maker. In group decision making processes, all individual decision maker's opinion is focused into a group opinion to construct an aggregated intuitionistic multi-fuzzy matrix $r_{i j}$ with the help of an IMFWA operator.

$$
\begin{aligned}
r_{i j}= & \operatorname{IMFWA}\left(r_{i j}^{(1)}, r_{i j}^{(2)}, \ldots, r_{i j}^{(L)}\right)=w_{1} R^{1} \oplus w_{2} R^{2} \oplus \ldots \oplus w_{L} R^{L} \\
& =\left\{1-\prod_{i=1}^{L} \prod_{j=1}^{k}\left(1-\mu_{R^{i}}^{j}\right) w^{w_{i}}, \prod_{i=1}^{L} \prod_{j=1}^{k}\left(v_{R^{i}}^{j}\right)^{w_{i}}, \prod_{i=1}^{L} \prod_{j=1}^{k}\left(1-\mu_{R^{i}}^{j}\right)^{w_{i}}-\prod_{i=1}^{L} \prod_{j=1}^{k}\left(v_{R^{i}}^{j}\right)^{w_{i}}\right\}
\end{aligned}
$$

The aggregated intuitionistic multi-fuzzy matrix can be defined as follows:

$$
R=\left[\begin{array}{cccc}
r_{11} & r_{12} \ldots & r_{1 n} \\
r_{21} & r_{22} \ldots & r_{2 n} \\
\cdot & \cdot & \cdot \\
r_{m 1} & r_{m 2} \ldots & \cdot \\
r_{m n}
\end{array}\right]
$$

where $r_{i j}=\left(\mu_{i j}^{(1)}, \mu_{i j}^{(2)}, \ldots, \mu_{i j}^{(k)}\right),\left(v_{i j}^{(1)}, v_{i j}^{(2)}, \ldots, v_{i j}^{(k)}\right),\left(\pi_{i j}^{(1)}, \pi_{i j}^{(2)}, \ldots, \pi_{i j}^{(k)}\right)$,

$$
\left.\mu_{i j}^{p}=1-\prod_{l=1}^{L}\left(1-\mu_{i j}^{l}\right)\right)^{w_{l}}, v_{i j}^{p}=\prod_{l=1}^{L}\left(v_{i j}^{l}\right)^{w_{l}}, \pi_{i j}^{p}=\prod_{l=1}^{L}\left(1-\mu_{i j}^{l}\right)^{w_{l}}-\prod_{l=1}^{L}\left(v_{i j}^{l}\right)^{w_{l}}, i \in M, j \in N, p \in k .
$$

Step 4: Distance matrices are constructed by deriving the normalized Hamming distance and Euclidean distance between the aggregated matrix $(\boldsymbol{R})$ and the sample matrix $(S)$. The distance matrix can be defined as follows: 
Table 3 Observation of expert I

\begin{tabular}{lccccc}
\hline Patients/symptoms & Chest pain & Palpitations & Dizziness & Fainting & Fatigue \\
\hline$P_{1}$ & $(0.3,0.7,0.5)$ & $(0.4,0.3,0.4)$ & $(0.1,0.2,0.0)$ & $(0.5,0.6,0.7)$ & $(0.4,0.3,0.4)$ \\
& $(0.2,0.1,0.4)$ & $(0.3,0.6,0.4)$ & $(0.7,0.7,0.8)$ & $(0.4,0.3,0.2)$ & $(0.6,0.4,0.4)$ \\
& $(0.5,0.2,0.1)$ & $(0.3,0.1,0.2)$ & $(0.2,0.1,0.2)$ & $(0.1,0.1,0.1)$ & $(0.0,0.3,0.2)$ \\
& $(0.4,0.3,0.5)$ & $(0.7,0.6,0.8)$ & $(0.6,0.5,0.4)$ & $(0.3,0.6,0.2)$ & $(0.4,0.7,0.5)$ \\
$P_{2}$ & $(0.5,0.4,0.4)$ & $(0.2,0.2,0.1)$ & $(0.3,0.3,0.4)$ & $(0.7,0.3,0.7)$ & $(0.1,0.2,0.3)$ \\
& $(0.1,0.3,0.1)$ & $(0.1,0.2,0.1)$ & $(0.1,0.2,0.2)$ & $(0.0,0.1,0.1)$ & $(0.5,0.1,0.2)$ \\
& $(0.1,0.2,0.1)$ & $(0.3,0.2,0.1)$ & $(0.8,0.7,0.8)$ & $(0.3,0.2,0.2)$ & $(0.4,0.3,0.2)$ \\
$P_{3}$ & $(0.7,0.6,0.9)$ & $(0.6,0.0,0.7)$ & $(0.0,0.1,0.1)$ & $(0.6,0.7,0.6)$ & $(0.4,0.7,0.7)$ \\
& $(0.2,0.2,0.0)$ & $(0.1,0.8,0.2)$ & $(0.2,0.2,0.1)$ & $(0.1,0.1,0.2)$ & $(0.2,0.0,0.1)$ \\
& $(0.5,0.4,0.5)$ & $(0.4,0.3,0.4)$ & $(0.2,0.1,0.0)$ & $(0.5,0.6,0.3)$ & $(0.4,0.5,0.4)$ \\
& $(0.4,0.4,0.3)$ & $(0.5,0.3,0.5)$ & $(0.7,0.6,0.7)$ & $(0.4,0.3,0.6)$ & $(0.6,0.4,0.3)$ \\
$P_{4}$ & $(0.1,0.2,0.2)$ & $(0.1,0.4,0.1)$ & $(0.1,0.3,0.3)$ & $(0.1,0.1,0.1)$ & $(0.0,0.1,0.3)$ \\
& & & & &
\end{tabular}

$$
T=\left[\begin{array}{cccc}
t_{11} & t_{12} & \cdots & t_{1 n} \\
t_{21} & t_{22} & \cdots & t_{2 n} \\
\vdots & \vdots & \ddots & \vdots \\
t_{m 1} & t_{m 2} & \cdots & t_{m n}
\end{array}\right]
$$

When both of $R$ and $S$ can be expressed as IMFM, the normalized Hamming distance $l^{\mathrm{H}}(R, S)$ and Euclidean distance $l^{\mathrm{E}}(R, S)$ over $R$ and $S$ in $U=\left(x_{1}, x_{2}, \ldots, x_{n}\right)$ of dimension $k$ can be defined with $t_{i j}$ where

$$
\begin{aligned}
t_{i j}^{\mathrm{H}} & =l^{\mathrm{H}}(R, S) \\
& =\frac{1}{2 n k} \sum_{j=1}^{n} \sum_{i=1}^{k}\left(\left|\mu_{R}^{i}\left(x_{j}\right)-\mu_{S}^{i}\left(x_{j}\right)\right|+\left|v_{R}^{i}\left(x_{j}\right)-v_{S}^{i}\left(x_{j}\right)\right|+\left|\pi_{R}^{i}\left(x_{j}\right)-\pi_{S}^{i}\left(x_{j}\right)\right|\right) .
\end{aligned}
$$

Table 4 Observation of expert II

\begin{tabular}{lccccc}
\hline Patients/symptoms & Chest pain & Palpitations & Dizziness & Fainting & Fatigue \\
\hline$P_{1}$ & $(0.5,0.6,0.4)$ & $(0.3,0.5,0.3)$ & $(0.4,0.6,0.6)$ & $(0.5,0.2,0.8)$ & $(0.4,0.7,0.8)$ \\
& $(0.2,0.2,0.5)$ & $(0.3,0.2,0.5)$ & $(0.2,0.3,0.2)$ & $(0.3,0.6,0.1)$ & $(0.4,0.0,0.1)$ \\
& $(0.3,0.2,0.1)$ & $(0.4,0.3,0.2)$ & $(0.2,0.1,0.2)$ & $(0.2,0.2,0.1)$ & $(0.2,0.3,0.1)$ \\
& $(0.6,0.2,0.7)$ & $(0.8,0.3,0.4)$ & $(0.3,0.3,0.3)$ & $(0.3,0.4,0.1)$ & $(0.5,0.3,0.4)$ \\
& $(0.3,0.4,0.2)$ & $(0.1,0.5,0.4)$ & $(0.6,0.4,0.3)$ & $(0.5,0.5,0.8)$ & $(0.3,0.5,0.5)$ \\
& $(0.1,0.4,0.1)$ & $(0.1,0.2,0.2)$ & $(0.1,0.3,0.4)$ & $(0.2,0.1,0.1)$ & $(0.2,0.2,0.1)$ \\
& $(0.6,0.6,0.8)$ & $(0.4,0.3,0.3)$ & $(0.6,0.4,0.3)$ & $(0.6,0.3,0.2)$ & $(0.3,0.4,0.2)$ \\
$P_{3}$ & $(0.2,0.3,0.1)$ & $(0.5,0.1,0.2)$ & $(0.2,0.4,0.4)$ & $(0.3,0.6,0.4)$ & $(0.3,0.4,0.6)$ \\
& $(0.2,0.1,0.1)$ & $(0.1,0.6,0.5)$ & $(0.2,0.2,0.3)$ & $(0.1,0.1,0.4)$ & $(0.4,0.2,0.2)$ \\
& $(0.5,0.6,0.7)$ & $(0.3,0.7,0.2)$ & $(0.2,0.3,0.2)$ & $(0.4,0.3,0.5)$ & $(0.2,0.4,0.3)$ \\
& $(0.3,0.2,0.1)$ & $(0.6,0.3,0.6)$ & $(0.5,0.3,0.5)$ & $(0.4,0.3,0.3)$ & $(0.5,0.4,0.4)$ \\
$P_{4}$ & $(0.2,0.2,0.2)$ & $(0.1,0.0,0.2)$ & $(0.3,0.4,0.3)$ & $(0.2,0.4,0.2)$ & $(0.3,0.2,0.3)$ \\
& & & & & \\
\end{tabular}


Table 5 Observation of expert III

\begin{tabular}{lccccc}
\hline Patients/symptoms & Chest pain & Palpitations & Dizziness & Fainting & Fatigue \\
\hline$P_{1}$ & $(0.3,0.4,0.7)$ & $(0.3,0.8,0.7)$ & $(0.4,0.1,0.2)$ & $(0.3,0.4,0.4)$ & $(0.1,0.3,0.7)$ \\
& $(0.5,0.5,0.3)$ & $(0.6,0.1,0.2)$ & $(0.3,0.5,0.8)$ & $(0.4,0.5,0.2)$ & $(0.7,0.3,0.3)$ \\
& $(0.2,0.1,0.0)$ & $(0.1,0.1,0.1)$ & $(0.3,0.4,0.0)$ & $(0.3,0.1,0.4)$ & $(0.2,0.4,0.0)$ \\
& $(0.6,0.4,0.5)$ & $(0.4,0.3,0.6)$ & $(0.4,0.6,0.5)$ & $(0.2,0.5,0.3)$ & $(0.7,0.5,0.3)$ \\
& $(0.2,0.3,0.4)$ & $(0.5,0.2,0.3)$ & $(0.3,0.3,0.4)$ & $(0.4,0.4,0.7)$ & $(0.2,0.2,0.3)$ \\
& $(0.2,0.3,0.1)$ & $(0.1,0.5,0.1)$ & $(0.3,0.1,0.1)$ & $(0.4,0.1,0.0)$ & $(0.1,0.3,0.4)$ \\
& $(0.4,0.2,0.6)$ & $(0.4,0.6,0.4)$ & $(0.6,0.5,0.3)$ & $(0.6,0.4,0.3)$ & $(0.3,0.3,0.3)$ \\
$P_{3}$ & $(0.3,0.6,0.2)$ & $(0.5,0.3,0.5)$ & $(0.3,0.4,0.6)$ & $(0.3,0.5,0.5)$ & $(0.4,0.6,0.5)$ \\
& $(0.3,0.2,0.2)$ & $(0.1,0.1,0.1)$ & $(0.1,0.1,0.1)$ & $(0.1,0.1,0.2)$ & $(0.3,0.1,0.2)$ \\
& $(0.8,0.6,0.7)$ & $(0.4,0.7,0.2)$ & $(0.6,0.4,0.4)$ & $(0.6,0.5,0.5)$ & $(0.3,0.4,0.4)$ \\
& $(0.1,0.1,0.3)$ & $(0.5,0.3,0.5)$ & $(0.3,0.4,0.3)$ & $(0.4,0.3,0.4)$ & $(0.5,0.4,0.3)$ \\
$P_{4}$ & $(0.1,0.3,0.0)$ & $(0.1,0.0,0.3)$ & $(0.1,0.2,0.3)$ & $(0.0,0.2,0.1)$ & $(0.2,0.2,0.3)$ \\
& & & & & \\
\hline
\end{tabular}

$$
\begin{aligned}
t_{i j}^{\mathrm{E}} & =l^{\mathrm{E}}(R, S) \\
& =\left(\frac{1}{2 n k} \sum_{j=1}^{n} \sum_{i=1}^{k}\left(\mu_{R}^{i}\left(x_{j}\right)-\mu_{S}^{i}\left(x_{j}\right)\right)^{2}+\left(v_{R}^{i}\left(x_{j}\right)-v_{S}^{i}\left(x_{j}\right)\right)^{2}+\left(\pi_{R}^{i}\left(x_{j}\right)-\pi_{S}^{i}\left(x_{j}\right)\right)^{2}\right)^{\frac{1}{2}}
\end{aligned}
$$

When $R$ is expressed as IMFM and $S$ can be expressed by a simple intuitionistic fuzzy matrix (IFM) which is the matrix representation of IFS in MCDM problems, normalized Hamming distance $l^{\mathrm{H}}(R, S)$ and Euclidean distance $l^{\mathrm{E}}(R, S)$ (the dimension of $R$ is $k$ and the dimension of $S$ is 1 ) can be defined with $t_{i j}$ where

$$
\begin{aligned}
t_{i j}^{\mathrm{H}} & =l^{\mathrm{H}}(R, S) \\
& =\frac{1}{2 n k} \sum_{j=1}^{n} \sum_{i=1}^{k}\left(\left|\mu_{R}^{i}\left(x_{j}\right)-\mu_{S}\left(x_{j}\right)\right|+\left|v_{R}^{i}\left(x_{j}\right)-v_{S}\left(x_{j}\right)\right|+\left|\pi_{R}^{i}\left(x_{j}\right)-\pi_{S}\left(x_{j}\right)\right|\right) .
\end{aligned}
$$

\begin{tabular}{|c|c|c|c|c|c|}
\hline Patients/symptoms & Chest pain & Palpitations & Dizziness & Fainting & Fatigue \\
\hline \multirow[t]{3}{*}{$\overline{P_{1}}$} & $(0.3,0.3,0.5)$ & $(0.6,0.4,0.4)$ & $(0.4,0.3,0.4)$ & $(0.6,0.3,0.4)$ & $(0.2,0.3,0.3)$ \\
\hline & $(0.2,0.1,0.4)$ & $(0.3,0.6,0.5)$ & $(0.4,0.7,0.4)$ & $(0.3,0.3,0.2)$ & $(0.7,0.5,0.3)$ \\
\hline & $(0.5,0.6,0.1)$ & $(0.1,0.0,0.1)$ & $(0.2,0.0,0.2)$ & $(0.1,0.4,0.4)$ & $(0.1,0.2,0.4)$ \\
\hline \multirow[t]{3}{*}{$P_{2}$} & $(0.3,0.3,0.5)$ & $(0.4,0.3,0.6)$ & $(0.4,0.5,0.6)$ & $(0.3,0.6,0.7)$ & $(0.3,0.2,0.4)$ \\
\hline & $(0.5,0.5,0.4)$ & $(0.5,0.2,0.3)$ & $(0.3,0.3,0.3)$ & $(0.5,0.3,0.1)$ & $(0.5,0.2,0.2)$ \\
\hline & $(0.2,0.2,0.1)$ & $(0.1,0.5,0.1)$ & $(0.3,0.2,0.1)$ & $(0.2,0.1,0.2)$ & $(0.2,0.6,0.4)$ \\
\hline \multirow[t]{3}{*}{$P_{3}$} & $(0.3,0.2,0.4)$ & $(0.3,0.2,0.4)$ & $(0.8,0.7,0.3)$ & $(0.3,0.7,0.1)$ & $(0.2,0.3,0.1)$ \\
\hline & $(0.2,0.6,0.1)$ & $(0.6,0.0,0.2)$ & $(0.0,0.1,0.1)$ & $(0.6,0.3,0.3)$ & $(0.2,0.2,0.1)$ \\
\hline & $(0.5,0.2,0.5)$ & $(0.1,0.8,0.4)$ & $(0.2,0.2,0.6)$ & $(0.1,0.0,0.6)$ & $(0.4,0.5,0.8)$ \\
\hline \multirow[t]{3}{*}{$P_{4}$} & $(0.2,0.4,0.3)$ & $(0.1,0.3,0.3)$ & $(0.2,0.1,0.1)$ & $(0.5,0.3,0.3)$ & $(0.4,0.3,0.0)$ \\
\hline & $(0.2,0.4,0.3)$ & $(0.5,0.3,0.2)$ & $(0.2,0.6,0.1)$ & $(0.4,0.3,0.1)$ & $(0.0,0.1,0.7)$ \\
\hline & $(0.6,0.2,0.4)$ & $(0.4,0.4,0.5)$ & $(0.6,0.3,0.8)$ & $(0.1,0.4,0.6)$ & $(0.6,0.6,0.3)$ \\
\hline
\end{tabular}

Table 6 Observation of expert IV 
Table 7 Symptoms vs diseases

\begin{tabular}{lcccc}
\hline Symptoms/stages & Stage I & Stage II & Stage III & Stage IV \\
\hline Chest pain & $(0.8,0.1,0.1)$ & $(0.7,0.2,0.1)$ & $(0.5,0.3,0.2)$ & $(0.1,0.7,0.2)$ \\
Palpitations & $(0.2,0.7,0.1)$ & $(0.5,0.1,0.4)$ & $(0.3,0.5,0.2)$ & $(0.3,0.6,0.1)$ \\
Dizziness & $(0.3,0.5,0.2)$ & $(0.4,0.2,0.4)$ & $(0.8,0.1,0.1)$ & $(0.5,0.1,0.4)$ \\
Fainting & $(0.5,0.3,0.2)$ & $(0.4,0.1,0.5)$ & $(0.3,0.2,0.5)$ & $(0.2,0.6,0.2)$ \\
Fatigue & $(0.5,0.4,0.1)$ & $(0.1,0.2,0.7)$ & $(0.4,0.4,0.2)$ & $(0.3,0.3,0.4)$ \\
\hline
\end{tabular}

$$
\begin{aligned}
t_{i j}^{\mathrm{E}} & =l^{\mathrm{E}}(R, S) \\
& =\left(\frac{1}{2 n k} \sum_{j=1}^{n} \sum_{i=1}^{k}\left(\mu_{R}^{i}\left(x_{j}\right)-\mu_{S}\left(x_{j}\right)\right)^{2}+\left(v_{R}^{i}\left(x_{j}\right)-v_{S}\left(x_{j}\right)\right)^{2}+\left(\pi_{R}^{i}\left(x_{j}\right)-\pi_{S}\left(x_{j}\right)\right)^{2}\right)^{\frac{1}{2}}
\end{aligned}
$$

Step 5: Distance matrices are searched to find out minimal distance for each alternative.

\section{Case study}

Statistics have consistently shown that heart disease is one of the leading causes of deaths in the USA and all over the world (CDC's report) [22]. Prompt and accurate diagnosis followed by proper treatment is necessary for life saving. Unfortunately, accurate diagnosis of heart diseases has never been an easy task. We illustrate the approach by using a medical diagnosis-based case study specifically for heart disease diagnosis. In this research work, we have categorized heart disease into four different stages based on a common set of symptoms like chest pain, palpitations, dizziness, fainting, and fatigue. Stage 'I' indicates that the patient is in the initial stages of heart disease and is fully curable if diagnosed at early stages and follows proper treatment. Patients who belong to stage 'II' are in more unsafe conditions and critical to cure. Stage 'III' is more unsafe in comparison to stage 'II', and the last stage is stage 'IV' wherein the disease is assumed to be not recoverable.

Let $D$ be the set of four related stages of heart disease (stage I, stage II, stage III, and stage IV) expressed by $D=\left\{d_{1}, d_{2}, d_{3}, d_{4}\right\}$ and $S$ be the set of five related symptoms

\begin{tabular}{|c|c|c|c|c|c|}
\hline Patients/symptoms & Chest pain & Palpitations & Dizziness & Fainting & Fatigue \\
\hline \multirow[t]{3}{*}{$\overline{P_{1}}$} & $(0.36,0.53,0.54)$ & $(0.41,0.55,0.48)$ & $(0.34,0.33,0.34)$ & $(0.49,0.39,0.62)$ & $(0.29,0.43,0.60)$ \\
\hline & $(0.25,0.18,0.39)$ & $(0.36,0.29,0.38)$ & $(0.36,0.52,0.48)$ & $(0.35,0.41,0.17)$ & $(0.59,0.00,0.24)$ \\
\hline & $(0.39,0.30,0.07)$ & $(0.23,0.16,0.15)$ & $(0.30,0.15,0.19)$ & $(0.17,0.20,0.22)$ & $(0.13,0.57,0.15)$ \\
\hline \multirow[t]{3}{*}{$P_{2}$} & $(0.49,0.30,0.56)$ & $(0.62,0.39,0.63)$ & $(0.44,0.49,0.46)$ & $(0.28,0.53,0.38)$ & $(0.50,0.46,0.40)$ \\
\hline & $(0.35,0.39,0.34)$ & $(0.27,0.25,0.24)$ & $(0.36,0.32,0.35)$ & $(0.51,0.37,0.44)$ & $(0.23,0.25,0.31)$ \\
\hline & $(0.16,0.30,0.10)$ & $(0.12,0.36,0.13)$ & $(0.21,0.19,0.19)$ & $(0.21,0.10,0.18)$ & $(0.27,0.29,0.29)$ \\
\hline \multirow[t]{3}{*}{$P_{3}$} & $(0.38,0.33,0.54)$ & $(0.35,0.35,0.31)$ & $(0.72,0.59,0.49)$ & $(0.47,0.44,0.20)$ & $(0.30,0.33,0.20)$ \\
\hline & $(0.30,0.50,0.21)$ & $(0.55,0.00,0.34)$ & $(0.00,0.20,0.22)$ & $(0.42,0.50,0.44)$ & $(0.31,0.43,0.38)$ \\
\hline & $(0.32,0.17,0.25)$ & $(0.10,0.65,0.35)$ & $(0.28,0.21,0.29)$ & $(0.10,0.06,0.36)$ & $(0.38,0.25,0.42)$ \\
\hline \multirow[t]{3}{*}{$P_{4}$} & $(0.55,0.51,0.58)$ & $(0.31,0.54,0.28)$ & $(0.33,0.24,0.19)$ & $(0.51,0.44,0.41)$ & $(0.33,0.40,0.29)$ \\
\hline & $(0.22,0.24,0.23)$ & $(0.52,0.30,0.42)$ & $(0.38,0.46,0.32)$ & $(0.40,0.30,0.29)$ & $(0.00,0.38,0.40)$ \\
\hline & $(0.23,0.25,0.19)$ & $(0.17,0.16,0.30)$ & $(0.29,0.31,0.49)$ & $(0.09,0.26,0.30)$ & $(0.67,0.31,0.31)$ \\
\hline
\end{tabular}

Table 8 Aggregated intuitionistic multi-fuzzy matrix 
Table 9 Distance matrix with equal weight $\left(w_{1}=w_{2}=w_{3}=w_{4}=1 / 4\right)$

\begin{tabular}{|c|c|c|c|c|c|c|c|c|c|}
\hline \multirow{2}{*}{$\begin{array}{l}\text { Patients/ } \\
\text { diseases }\end{array}$} & \multicolumn{4}{|c|}{ Hamming distance } & \multicolumn{4}{|c|}{ Euclidean distance } & \multirow{2}{*}{$\begin{array}{c}\text { Diagnosis } \\
\text { result }\end{array}$} \\
\hline & Stage I & Stage II & Stage III & Stage IV & Stage I & Stage II & Stage III & Stage IV & \\
\hline$P_{1}$ & 0.23 & 0.31 & 0.28 & 0.34 & 0.25 & 0.30 & 0.28 & 0.32 & Stage I \\
\hline$P_{2}$ & 0.26 & 0.30 & 0.25 & 0.27 & 0.27 & 0.29 & 0.24 & 0.26 & Stage III \\
\hline$P_{3}$ & 0.32 & 0.31 & 0.23 & 0.24 & 0.32 & 0.29 & 0.24 & 0.26 & Stage III \\
\hline$P_{4}$ & 0.23 & 0.26 & 0.25 & 0.29 & 0.23 & 0.25 & 0.28 & 0.30 & Stage I \\
\hline
\end{tabular}

(chest pain, palpitations, dizziness, fainting, and fatigue) given by $S=\left\{s_{1}, s_{2}, s_{3}, s_{4}, s_{5}\right\}$. Suppose that a group of four experts $E=\left\{e_{1}, e_{2}, e_{3}, e_{4}\right\}$ is monitoring the symptoms as per their knowledgebase to reach an agreement about which stage of heart disease is more likely to appear for a particular patient. A set of four patients $P=\left\{P_{1}, P_{2}, P_{3}, P_{4}\right\}$ is considered in this study. This article assumes that the set of experts $\left\{e_{1}, e_{2}, e_{3}, e_{4}\right\}$ provides their opinions on a set of symptoms $\left\{s_{1}, s_{2}, s_{3}, s_{4}, s_{5}\right\}$ by means of intuitionistic multi-fuzzy sets $M=\left\{M^{1}, M^{2}, M^{3}, M^{4}\right\}$ as depicted below (Tables 3, 4, 5, and 6). Table 7 shows the reference values of each of the symptoms for the mentioned stages (stage I, stage II, stage III, and stage IV) of heart disease. The aggregated intuitionistic multifuzzy decision matrix based on the opinions of decision makers is shown in Table 8 .

As the patient's condition and variations of symptoms differ with time, for an expert, it might not be feasible to make a decision based on one inspection of the patient. A probable solution might be to inspect the patient at regular time intervals and record them accordingly. This paper has taken the sample readings at three different time intervals of a day. So each symptom is described by three membership functions $(\mu)$, three non-membership functions $(v)$, and three hesitation margins $(\pi)$.

In accordance with the case study, the various steps of the algorithm are described as follows:

Step 1: Intuitionistic multi-fuzzy matrix $R=\left\{R^{1}, R^{2}, R^{3}, R^{4}\right\}$ for each expert $E=\left\{e_{1}, e_{2}\right.$, $\left.e_{3}, e_{4}\right\}$ is constructed by considering the three consecutive observations of those experts. Tables 3, 4, 5, and 6 shows the intuitionistic multi-fuzzy decision matrices $\left(R^{1}, R^{2}, R^{3}, R^{4}\right)$ for expert I $\left(e_{1}\right)$, expert II $\left(e_{2}\right)$, expert III $\left(e_{3}\right)$, and expert IV $\left(e_{4}\right)$, respectively.

Step 2: In the case study, we have considered two real cases. Initially, the weights of each expert are taken to be equal. In the second case, we assume that the weights of each expert are different. Due to experts' variation in experience and domain of expertise, different priorities can be assigned to different experts, so variations in their weights during the decision making process is practical.

Step 3: An aggregated intuitionistic multi-fuzzy matrix is constructed based on the opinions of the decision makers. When the weights of each expert are equal, Table 8 shows the corresponding aggregated intuitionistic multi-fuzzy matrix.

Table 10 Distance matrix with different weights $\left(w_{1}=0.1, w_{2}=0.2, w_{3}=0.3, w_{4}=0.4\right)$

\begin{tabular}{|c|c|c|c|c|c|c|c|c|c|}
\hline \multirow{2}{*}{$\begin{array}{l}\text { Patients/ } \\
\text { diseases }\end{array}$} & \multicolumn{4}{|c|}{ Hamming distance } & \multicolumn{4}{|c|}{ Euclidean distance } & \multirow{2}{*}{$\begin{array}{c}\text { Diagnosis } \\
\text { result }\end{array}$} \\
\hline & Stage I & Stage II & Stage III & Stage IV & Stage I & Stage II & Stage III & Stage IV & \\
\hline$\overline{P_{1}}$ & 0.24 & 0.31 & 0.27 & 0.34 & 0.26 & 0.30 & 0.27 & 0.31 & Stage I \\
\hline$P_{2}$ & 0.26 & 0.29 & 0.23 & 0.26 & 0.26 & 0.28 & 0.23 & 0.26 & Stage III \\
\hline$P_{3}$ & 0.34 & 0.29 & 0.26 & 0.25 & 0.34 & 0.28 & 0.26 & 0.25 & Stage IV \\
\hline$P_{4}$ & 0.26 & 0.24 & 0.26 & 0.30 & 0.26 & 0.24 & 0.28 & 0.30 & Stage ॥ \\
\hline
\end{tabular}


Table 11 Distance matrix with different weight $\left(w_{1}=0.3, w_{2}=0.2, w_{3}=0.4, w_{4}=0.1\right)$

\begin{tabular}{|c|c|c|c|c|c|c|c|c|c|}
\hline \multirow{2}{*}{$\begin{array}{l}\text { Patients/ } \\
\text { diseases }\end{array}$} & \multicolumn{4}{|c|}{ Hamming distance } & \multicolumn{4}{|c|}{ Euclidean distance } & \multirow{2}{*}{$\begin{array}{c}\text { Diagnosis } \\
\text { result }\end{array}$} \\
\hline & Stage I & Stage II & Stage III & Stage IV & Stage I & Stage II & Stage III & Stage IV & \\
\hline$P_{1}$ & 0.23 & 0.32 & 0.29 & 0.35 & 0.25 & 0.31 & 0.30 & 0.33 & Stage I \\
\hline$P_{2}$ & 0.27 & 0.31 & 0.25 & 0.27 & 0.27 & 0.30 & 0.25 & 0.27 & Stage III \\
\hline$P_{3}$ & 0.31 & 0.34 & 0.22 & 0.24 & 0.31 & 0.32 & 0.23 & 0.25 & Stage III \\
\hline$P_{4}$ & 0.19 & 0.27 & 0.26 & 0.30 & 0.21 & 0.27 & 0.28 & 0.31 & Stage I \\
\hline
\end{tabular}

Steps 4 and 5: This step calculates distance matrices using normalized Hamming distance and normalized Euclidean distance for each patient for the set of diseases. The lowest distance shows proper diagnosis in both Hamming distance and Euclidean distance measurements. This step is illustrated in two phases: in the first phase, all decision makers are assumed to have equal importance or weight $\left(w_{1}=w_{2}=w_{3}=w_{4}=1 / 4\right)$, and the next phase considers different weights according to the priority of each individual decision makers. Table 9 presents the outcome of the first phase, and Tables 10, 11, and 12 present the outcomes of the second phase. Each table shows the result of Hamming distance measurements, Euclidean distance measurements, and diagnostic outcome. Tables 9, 10, 11, and 12 are constructed using Table 7 (sample matrix) and Table 8 (aggregated matrix) and the expressions (18) and (19).

Diagnostic results from Table 9 indicates that patient $P_{1}$ and $P_{4}$ are in stage I, while $P_{2}$ and $P_{3}$ are in stage III which is similar to the diagnostic result of Table 11. Other diagnostic results from Table 10 specifies that patient $P_{1}$ is in stage I, $P_{2}$ is in stage III, $P_{3}$ is in stage IV, and $P_{4}$ is in stage II which is similar to Table 12. Hamming distance and Euclidean distance measurements are observed to produce similar diagnostic outcome in all the cases.

When experts' opinions (IMFS) are degenerated to IFS as per subsection 'Presentation of IMFS to IFS degeneration', the various distance measurements results are given in Tables 13 and 14 for similar weight experts and different weight experts, respectively. From the given result set, it can be observed that the diagnostic result of Table 13 is similar with that of Table 9, but the result of Table 14 is different from that of Table 10. This study indicates that when weights of individual experts are equal, a similar diagnostic outcome can be found in both cases (IMFS and degenerated IFS), but when weights of experts are different, then different diagnostic outcomes can be found. In general, the diagnostic outcome is different when IMFS is degenerated to IFS. This happens due to multiple-time inspection of experts in the case of IFMS and one-time inspection for IFS.

Table 12 Distance matrix with different weight $\left(w_{1}=0.2, w_{2}=0.1, w_{3}=0.3, w_{4}=0.4\right)$

\begin{tabular}{|c|c|c|c|c|c|c|c|c|c|}
\hline \multirow{2}{*}{$\begin{array}{l}\text { Patients/ } \\
\text { diseases }\end{array}$} & \multicolumn{4}{|c|}{ Hamming distance } & \multicolumn{4}{|c|}{ Euclidean distance } & \multirow{2}{*}{$\begin{array}{l}\text { Diagnosis } \\
\text { result }\end{array}$} \\
\hline & Stage I & Stage II & Stage III & Stage IV & Stage I & Stage II & Stage III & Stage IV & \\
\hline$\overline{P_{1}}$ & 0.24 & 0.31 & 0.29 & 0.35 & 0.26 & 0.31 & 0.29 & 0.32 & Stage I \\
\hline$P_{2}$ & 0.26 & 0.29 & 0.24 & 0.26 & 0.27 & 0.28 & 0.23 & 0.26 & Stage III \\
\hline$P_{3}$ & 0.35 & 0.31 & 0.25 & 0.23 & 0.35 & 0.29 & 0.25 & 0.25 & Stage IV \\
\hline$P_{4}$ & 0.26 & 0.25 & 0.26 & 0.30 & 0.25 & 0.24 & 0.28 & 0.30 & Stage ॥ \\
\hline
\end{tabular}


Table 13 Distance matrix with equal weight $\left(w_{1}=w_{2}=w_{3}=w_{4}=1 / 4\right)$

\begin{tabular}{|c|c|c|c|c|c|c|c|c|c|}
\hline \multirow{2}{*}{$\begin{array}{l}\text { Patients/ } \\
\text { diseases }\end{array}$} & \multicolumn{4}{|c|}{ Hamming distance } & \multicolumn{4}{|c|}{ Euclidean distance } & \multirow{2}{*}{$\begin{array}{c}\text { Diagnosis } \\
\text { result }\end{array}$} \\
\hline & Stage I & Stage II & Stage III & Stage IV & Stage I & Stage II & Stage III & Stage IV & \\
\hline$P_{1}$ & 0.17 & 0.31 & 0.20 & 0.29 & 0.33 & 0.50 & 0.41 & 0.49 & Stage I \\
\hline$P_{2}$ & 0.26 & 0.29 & 0.22 & 0.25 & 0.44 & 0.49 & 0.40 & 0.42 & Stage III \\
\hline$P_{3}$ & 0.30 & 0.29 & 0.18 & 0.20 & 0.49 & 0.46 & 0.32 & 0.36 & Stage III \\
\hline$P_{4}$ & 0.18 & 0.28 & 0.21 & 0.26 & 0.31 & 0.45 & 0.43 & 0.48 & Stage I \\
\hline
\end{tabular}

\section{Conclusion}

In this paper, we first present an intuitionistic multi-fuzzy set which is a combination of an intuitionistic fuzzy set and a multi-fuzzy set. We then introduce some basic operations on intuitionistic multi-fuzzy set and their properties. Especially, we introduce the concept of intuitionistic multi-fuzzy weighted averaging operator which can be treated as an extension of intuitionistic weighted arithmetic mean operator. We have proposed an algorithmic approach which combines the opinions of individual decision makers to produce an aggregated matrix by using a group multi-criteria decision making method. In the proposed approach, we measure the Hamming distance and Euclidean distance of each patient for the set of diseases by considering the symptoms of that particular disease where both type of distance measurements yield similar diagnostic result. The lowest distance shows proper diagnosis for both Hamming distance and Euclidean distance measurements. The observed case study illustrates our approach in two phases: in the first phase, all decision makers are assumed to have equal importance or weight, and the next phase considers different weights for each individual decision makers. Our observation proves that changes in experts' weight might have several impacts on disease diagnosis, i.e., diagnosis might vary for different patients. In this study, we also present score function and accuracy function in the context of IMFS with an application to MCDM. Finally, this study degenerates the individual experts' opinions (IMFS) into IFS and compares the final outcome which is found to be different. The concept of multiplicity is incorporated by taking samples from the same patient at different times. A future scope of this research work might be enhancing the study of intuitionistic multi-fuzzy set for uncertain group decision making problems where GDM is crucial due to the lack of information, expertise of the experts, risk amendment, etc. An example of these type of problems might be critical disease diagnosis, risk management, natural disaster forecasting, typical financial decision making, etc. Researchers may introduce the advantages of machine learning techniques to allow updating of information for decision makers which would be more adaptable in the real life environments.

Table 14 Distance matrix with different weights $\left(w_{1}=0.1, w_{2}=0.2, w_{3}=0.3, w_{4}=0.4\right)$

\begin{tabular}{|c|c|c|c|c|c|c|c|c|c|}
\hline \multirow{2}{*}{$\begin{array}{l}\text { Patients/ } \\
\text { diseases }\end{array}$} & \multicolumn{4}{|c|}{ Hamming distance } & \multicolumn{4}{|c|}{ Euclidean distance } & \multirow{2}{*}{$\begin{array}{l}\text { Diagnosis } \\
\text { result }\end{array}$} \\
\hline & Stage I & Stage II & Stage III & Stage IV & Stage I & Stage II & Stage III & Stage IV & \\
\hline$\overline{P_{1}}$ & 0.18 & 0.31 & 0.20 & 0.29 & 0.35 & 0.50 & 0.39 & 0.48 & Stage I \\
\hline$P_{2}$ & 0.25 & 0.28 & 0.21 & 0.24 & 0.42 & 0.47 & 0.37 & 0.41 & Stage III \\
\hline$P_{3}$ & 0.31 & 0.26 & 0.20 & 0.21 & 0.50 & 0.41 & 0.34 & 0.37 & Stage III \\
\hline$P_{4}$ & 0.21 & 0.25 & 0.22 & 0.25 & 0.35 & 0.40 & 0.42 & 0.47 & Stage I \\
\hline
\end{tabular}




\section{Acknowledgements}

The authors wish to express sincere gratitude to the anonymous reviewers for their constructive comments and helpful suggestions, which led to substantial improvements of this paper.

\section{Author details}

${ }^{1}$ Department of CSE, Dr. B. C. Roy Engineering College, Durgapur, West Bengal 713206, India. ${ }^{2}$ Department of CSE, Heritage Institute of Technology, Kolkata, West Bengal 700107, India. ${ }^{3}$ Department of Mathematics, National Institute of Technology, Durgapur, West Bengal 713209, India.

Received: 18 April 2013 Accepted: 10 October 2013

Published: 7 November 2013

\section{References}

1. Atanassov, K: Intuitionistic fuzzy sets. Springer Physica, Heidelberg (1999)

2. Zadeh, LA: Fuzzy sets. Inf. Control. 8, 338-356 (1965)

3. Chen, SM, Tan, JM: Handling multi criteria fuzzy decision-making problems based on vague set theory. Fuzzy Set. Syst. 67, 163-172 (1994)

4. Hong, DH, Choi, CH: Multi criteria fuzzy decision-making problems based on vague set theory. Fuzzy Set. Syst. $114,103-113(2000)$

5. Li, DF: Multi attribute decision making models and methods using intuitionistic fuzzy sets. J. Comput. Syst. Sci. 70, 73-85 (2005)

6. Li, F, Lu, A, Cai, L: Methods of multi-criteria fuzzy decision making based on vague sets. J. Huazhong. Univ. Sci. Technolog. Med. Sci. 29, 1-3 (2001)

7. Li, F, Rao, Y: Weighted methods of multi-criteria fuzzy decision making based on vague sets. Comput. Sci. $28,60-65(2001)$

8. Lin, L, Yuan, XH, Xia, ZQ: Multi criteria fuzzy decision-making methods based on intuitionistic fuzzy sets. J. Comput. Syst. Sci. 73, 84-88 (2007)

9. Liu, HW, Wang, GJ: Multi-criteria decision-making methods based on intuitionistic fuzzy sets. Eur. J. Oper. Res. $179,220-233(2007)$

10. Atanassov, K, Pasi, G, Yager, R: Intuitionistic fuzzy interpretations of multi-person multicriteria decision making Proc. of 2002 First Intern. IEEE Symposium Intell. Syst. 1, 115-119 (2002)

11. Atanassov, K, Pasi, G, Yager, R, Atanassova, V: Intuitionistic fuzzy graph interpretations of multi-person multi-criteria decision making. In: Proceedings of the Third Conference of the European Society for Fuzzy Logic and Technology EUSFLAT, Zittau, 10-12 September 2003, pp. 177-182

12. Pasi, G, Atanassov, K, Melo Pinto, P, Yager, R, Atanassov, V: Multi-person multi-criteria decision making: intuitionistic fuzzy approach and generalized net model. In: Advanced design, production and management systems. Proceedings of the 10th ISPE International Conference on Concurrent Engineering, Madeira, 26-30 July 2003, pp. 1073-1078

13. Blizard, WD: Multiset theory. Notre Dame J. Formal Logic 30(1), 36-66 (1989)

14. Yager, RR: On the theory of bags. Int. J. Gen. Syst. 13, 23-37 (1986)

15. Goguen, JA: L-fuzzy sets. J. Math. Anal. Appl. 18, 145-174 (1967)

16. Atanassov, KT: Intuitionistic fuzzy sets. Fuzzy Set. Syst. 20, 87-96 (1986)

17. Yager, RR: On ordered weighted averaging aggregation operators in multi criteria decision making. IEEE Trans. Syst. Man Cybern. 18, 183-190 (1988)

18. De, SK, Biswas, R, Roy, AR: An application of intuitionistic fuzzy sets in medical diagnosis. Fuzzy Set. Syst. 117(2), 209-213 (2001)

19. Atanassov, K: New operations defined over the intuitionistic fuzzy sets. Fuzzy Set. Syst. 61, 137-142 (1994)

20. Dey, SK, Biswas, R, Roy, AR: Some operations on intuitionistic fuzzy sets. Fuzzy Set. Syst. 114, 477-484 (2000)

21. $\mathrm{Xu}, \mathrm{Z}$ : Intuitionistic preference relations and their application in group decision making. Inform. Sci. $177,2363-2379(2007)$

22. CDC report: http://www.cdc.gov/nccdphp/overview.htm Accessed 13 Aug 2012

doi:10.1186/2195-5468-1-10

Cite this article as: Das et al:: Group multi-criteria decision making using intuitionistic multi-fuzzy sets.

Journal of Uncertainty Analysis and Applications 2013 1:10.

\section{Submit your manuscript to a SpringerOpen ${ }^{\circ}$} journal and benefit from:

- Convenient online submission

- Rigorous peer review

- Immediate publication on acceptance

- Open access: articles freely available online

- High visibility within the field

- Retaining the copyright to your article

Submit your next manuscript at $\boldsymbol{\sim}$ springeropen.com 